\title{
A flexible, on-line magnetic spectrometer for ultra-intense laser produced fast electron measurement
}

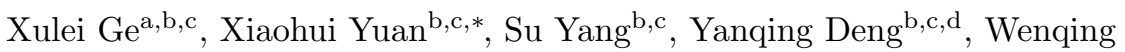 \\ Wei ${ }^{\mathrm{b}, \mathrm{c}}$, Yuan Fang ${ }^{\mathrm{b}, \mathrm{c}}$, Jian Gao ${ }^{\mathrm{b}, \mathrm{c}}$, Feng Liu ${ }^{\mathrm{b}, \mathrm{c}, * *}$, Min Chen ${ }^{\mathrm{b}, \mathrm{c}}, \mathrm{Li}_{\mathrm{Zhao}}^{\mathrm{a}}$, \\ Yanyun $\mathrm{Ma}^{\mathrm{c}, \mathrm{d}}$, Zhengming Sheng ${ }^{\mathrm{b}, \mathrm{c}, \mathrm{e}}$, Jie Zhang ${ }^{\mathrm{b}, \mathrm{c}}$ \\ ${ }^{a}$ State Key Laboratory of Surface Physics and Department of Physics, Fudan University, \\ Shanghai 200433, China \\ ${ }^{b}$ Key Laboratory for Laser Plasmas (Ministry of Education) and School of Physics and \\ Astronomy, Shanghai Jiao Tong University, Shanghai 200240, China \\ ${ }^{c}$ Collaborative Innovation Center of IFSA (CICIFSA), Shanghai Jiao Tong University, \\ Shanghai 200240, China \\ ${ }^{d}$ College of Science, National University of Defense Technology, Changsha 410073, China \\ ${ }^{e}$ SUPA, Department of Physics, University of Strathclyde, Glasgow G4 ONG, UK
}

\begin{abstract}
We have developed an on-line magnetic spectrometer to measure energy distributions of fast electrons generated from ultra-intense laser-solid interactions. The spectrometer consists of a sheet of plastic scintillator, a bundle of nonscintillating plastic fibers, and an sCMOS camera recording system. The design advantages include on-line capturing ability, versatility of detection arrangemen$t$, and resistance to harsh in-chamber environment. The validity of the instrument was tested experimentally. This spectrometer can be applied to the characterization of fast electron source for understanding fundamental laser-plasma interaction physics and to the optimization of high-repetition-rate laser-driven applications.
\end{abstract}

Keywords: electron spectrometer; on-line spectrometer; ultra-intense laser-solid interaction

\footnotetext{
* Corresponding author: Xiaohui Yuan

${ }^{* *}$ Corresponding author: Feng Liu

Email addresses: xiaohui.yuan@sjtu.edu.cn (Xiaohui Yuan), liuf001@sjtu.edu.cn (Feng Liu)
} 


\section{Introduction}

The interaction of ultra-intense laser with overdense plasma produces largecurrent relativistic (fast) electron beams. Knowing the electron energy distribution and its dependence on laser and target parameters are important to the

5 understanding of fundamental laser-plasma physics, and to the optimization of electron sources for applications, e.g., the fast ignition (FI)[1] approach to inertial confinement fusion (ICF), laser-driven ion acceleration $[2,3]$ and bright radiation sources development $[4,5]$.

The electron energy spectrum is normally measured with a dipole magnet based electron spectrometer. The electrons are first dispersed according to the different kinetic energies in the magnetic field and then recorded by the detector(s). Three major detection methods previously reported are image plates (IPs), scintillating fibers and scintillator screen both imaged directly with C$\mathrm{CD} / \mathrm{sCOMS}$ camera. The major drawback of $\operatorname{IPs}[6,7]$ is the need to vent the 15 vacuum chamber to read the stored signals. Therefore IPs are not appropriate for high-repetition-rate experiments. The scintillator fibers[8-11] are normally placed parallel to the magnetic lines along the sides of both plates. The effects of fringe field on electron deflection trajectory are inevitable[12]. In addition, the effective fiber length which produces scintillating light is only a small portion of the fiber. The longer redundant fibers beyond the magnetic field region will produce background scintillating light when exposed to optical light or particles. To eliminate the undesired background, Chen et al[10] has put the CCD camera inside the vacuum chamber to shorten the fiber length. However, the CCD suffered from the strong electromagnetic pulses (EMP)[13] in the harsh environment and may not work properly[14]. When scintillator screens[15-18] were used, they were normally imaged directly with an optical lens coupled CCD camera. Extensive shielding is an essential requirement to avoid scattered optical light entering the imaging system. Due to the large aspect ratio of screen (> 10) and considerable length along the dispersion direction (normally tens so of centimeters), effectively only a very small portion of CCD chip was used, 
limiting the spatial resolution on the scintillator detector by the imaging system to only several hundred microns[2]. Another important issue is that the imaging system needs be realigned when changing the positions of the electron spectrometer to measure electron spectra along other directions.

In this paper, we present a novel design of a flexible, on-line magnetic spectrometer, which utilizes the scintillator screen to produce scintillating light and employs the non-scintillating fibers to guide light to high frame-rate sCMOS camera imaging system. This instrument combines the advantages of the online capability of scintillator, flexibility and versatile transforming configuration of the fibers, and resistance to harsh environment by moving the recording electronics out of the vacuum chamber.

\section{Spectrometer design}

The schematic of the spectrometer is shown in Fig. 1(a) (the imaging system is not presented). A pair of parallel $\mathrm{NdFeB}$ permanent magnets, shown

45 in light blue, deflects the electrons according to their different kinetic energies (representative lines in black). A thin sheet of plastic scintillator (in dark red) is inserted inside the magnetic spectrometer to convert the electron signal into visible optical light. The scintillator screen can facilitate an on-line detection capability since the emitted light from the scintillator can be captured by the imaging system without disturbing the detector. This would attract more interests especially in high-repetition-rate single-shot evaluations for statistical analysis.

A bundle of fibers, arranged in multiple rows of linear arrays, are placed with one end in close contact with the scintillator sheet. The light signals from the scintillator are then relayed by the fibers to the imaging system placed outside the vacuum chamber[19]. A high frame-rate sCMOS camera located outside the vacuum chamber is used to record the optical light transmitted through a glass window. Considering the aspect ratio of sCMOS chip size, the other end of the fiber bundle is rearranged to an areal configuration. The insets in Fig. 1 
(a) illustrate the linear and areal interfaces of the fiber bundle, respectively. A photo of the assembly is shown in Fig. 1 (b).

Trapezoidal-shaped (truncated square) magnets (marked by dashed-line in Fig. 2 (a)) are used in this work. The magnet plates have an overall side-length of $200 \mathrm{~mm}$ and a separation gap of $10 \mathrm{~mm}$. They are enclosed with a 10-mm65 thick iron yoke. The spatial distribution of the magnetic field strength in the mid-plane was mapped out with a Hall probe, and is shown as the grayed area in Fig. 2(a). The peak field strength is $0.12 \mathrm{~T}$. The fringed field decreases from $0.10 \mathrm{~T}$ at the edge to $0.05 \mathrm{~T} 10 \mathrm{~mm}$ away from the magnet plates. If the detector is not inside the uniform region and there is no collimator at the entrance, the electron trajectories and energy dispersions need to be precisely simulated using a particle tracing program[12] in order to include the effects of fringe fields. Simplified calculations using simple lorentz force consideration can be obtained by minimizing fringe field effects with a collimator placed inside a central region of $160 \mathrm{~mm}$ with field strength variation of only $1.26 \%$ (standard deviation).

75 An ultralow-carbon stainless-steel collimator was chosen as the entrance. The collimator is $30 \mathrm{~mm}$ long with a $3.5 \mathrm{~mm}$ hole in the center (yellow in Fig. 2(a)). The magnetic field increases from 0 to 0.12 Tesla within $13 \mathrm{~mm}$ beyond the collimator hole. The scintillator screen was also inserted into the near-uniform magnetic field region further reducing the effect of fringe field. A visible CW so laser (red dash line) was used to align the spectrometer.

The plastic scintillator is a DRZ fluorescent screen $\left(\mathrm{Gd}_{2} \mathrm{O}_{2} \mathrm{~S}\right.$ : Tb, type $\left.\mathrm{Pl}-200\right)$ having dimensions of $200 \mathrm{~mm}$ (length) $\times 10 \mathrm{~mm}$ (width) $\times 630 \mu \mathrm{m}$ (thickness) and emits light at $546 \mathrm{~nm}$ when impacted by energetic electrons. The scintillator screen itself has a higher spatial resolution (a few 10's of microns) than the largediameter scintillator fibers (typical $500 \mu \mathrm{m}$ )[10] and it is not the limiting factor for the energy resolution. A $2 \mu m$-thick aluminum-anodized mylar film was placed before the scintillator to block scatted light. This type of scintillator has been calibrated using ultrashort electron beam from a radio frequency linear electron accelerator[20]. The $20 \mathrm{~cm}$-long plastic scintillator sheet (dark-red line 90 in Fig. 1(a)) was positioned at $45^{\circ}$ with respect to the entrance axis. In this case, 

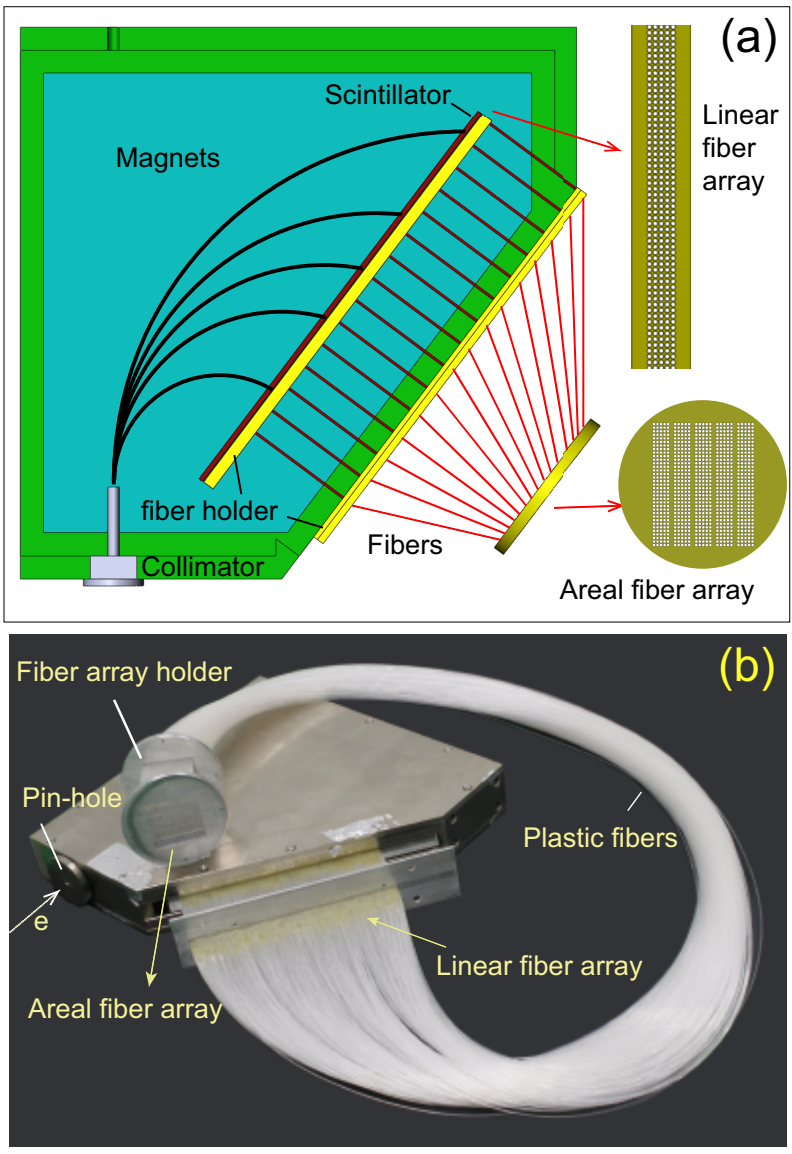

Figure 1: (color online) The schematic (a) and photograph (b) of the magnetic spectrometer. The regions in light blue and in green are magnet plates and the yoke, respectively. A collimator was inserted into the region of near-uniform magnetic field strength. The red lines indicate the fiber bundles. Two insets in (a) show the linear and areal interfaces of the fiber bundle. 
the spectrometer works in the focusing mode, i.e. finite-diameter collimated electron beam is bent by the magnet and overlaps on the detector plane[11]. The corresponding trajectories of the electrons with different energies were computed by calculating the deflection angle according to the Lorentz force, which are shown in Fig. 2(a).

A total number of 875 equally spaced 1-meter-long plastic fibers are placed in close contact with the scintillator, to sample and guide the optical light signals out of the spectrometer. The fiber bundle was further wrapped with aluminum to avoid stray light. The cross-talk between channels was also checked using a point laser source to be $<4 \%$. Although the spatial resolution of the nonscintillating fibers is not as high as that of IPs, they were chosen due to several advantages. Firstly, it is easier to shield the imaging system from scattered light$\mathrm{s}$, and no optics are required in the crowded chamber, saving space for other diagnostics. Secondly, the fibers can be easily bent. Therefore, the imaging system can be setup using chamber windows away from the influences of radiations (especially EMPs) and also to avoid conflicts with other diagnostics. Thirdly, the plastic fibers can be rearranged into a matrix to fit the sCMOS/CCD camera sensor for better imaging. Fourthly, the imaging component does not require alignment, when re-positioning of the main body of the spectrometer becomes necessary to measure the electron spectra along other directions. Lastly, the fibers are insensitive to the radiations, hence no extensive shieldings are needed.

Each fiber has a diameter of $0.75 \mathrm{~mm}$. The separation of the centers of adjacent fibers is $1 \mathrm{~mm}$. In the scintillator end, five fibers were employed in the non-dispersion direction for each specific electron energy, thus forming a matrix of $5 \times 175$ fibers. This gives rise to 175 energy points in the dispersion direction. Figure 2(b) shows the energy dispersion and the resolving power $(E / \Delta E)$ with respect to the electron energy $(E)$, where $\Delta E$ is the energy resolution at $E$. The electron traces in the magnetic field could be calculated using ${ }_{120}$ equation $d(\gamma m \vec{v}) / d t=-e \cdot \vec{v} \times \vec{B} / c$. Where $\gamma$ is the relativity factor, $m$ is the electron mass, $e$ is the electronic charge, $\vec{B}$ is the magnetic field, $\vec{v}$ is the 
speed of electrons and $c$ is the speed of light. In this case, E is calculated to be ranging from $0.5 \mathrm{MeV}$ to $4.8 \mathrm{MeV}$. The energy resolutions of the spectrometer are determined by diameter of fibers and the beam sizes on scintillator. The typical electron trajectories in the energy range of 1-4.5 MeV for an acceptance angle of $2.4 \times 10^{-4} \mathrm{sr}$ are shown in Fig. 2(a). The trajectories of mono-energetic electrons entrancing the magnetic at two edges of the collimator will crossed on the scintillator at two different positions as shown in Fig. 2(a). The distance between these two positions would be the beam size on the scintillator and it is marked by the size of the black dots in Fig. 2(a). The beam sizes on the scintillator (black dots) are determined by the electron divergence, the acceptance angle of the collimator, electron energies, magnetic field and the position of scintillator. It is less than $0.75 \mathrm{~mm}$ for energies lower than $1.4 \mathrm{MeV}$. For electrons with energies higher than $1.4 \mathrm{MeV}$, the beam size is larger than the fiber diameter, which becomes a predominant factor limiting the energy resolution. As shown in Fig. 2(b), the resolving power first increases from 30 to 77 for the electron energies of $0.5-1.4 \mathrm{MeV}$ due to the fiber diameter and then decreases to 57 from $1.4 \mathrm{MeV}$ to $4.8 \mathrm{MeV}$ when the beam sizes are bigger than fiber diameter. Since smaller diameter optical fibers (a few 10's of microns) are available, decreasing the acceptance angle or the diameter of the fibers will improve the energy resolution.

The other end of the fiber bundle is in close contact with a thin glass window. Due to the fiber flexibility, the window could be selected from any flange on the chamber, preferably away from the laser incident plane. A 16-bit sCMOS camera (ORCA-Flash4.0 LT, from Hamamatsu Photonics) coupled with a $\mathrm{f}=6-12 \mathrm{~mm} / \mathrm{F} 1.6$ lens was used to image the fibers. The sCMOS camera was chosen with consideration of several superior advantages: small pixel size (6.5 $\mu \mathrm{m})$, high frame rate and low cost while remaining comparable in quantum efficiency to EMCCD. The fiber bundle was re-organised to an areal configuration of $25 \times 35$ fibers to maximize the usage of the sCMOS chip such that the resolution of the imaging system is $\sim 50 \mu \mathrm{m} /$ pixel. Similar work was reported by Kodama et al that two-dimensional fibers were rearranged to a line to fit a 
streak camera[21]. The light transport efficiency of the fiber bundle and imaging system was relatively calibrated and the variation between energy channels was $4.5 \%$ (standard deviation). Since the camera was detached from the target chamber, the shielding was much easier.

\section{Experiment and results}

The experiment was carried out using the $200 \mathrm{TW} \mathrm{Ti}$ : sapphire laser at Shanghai Jiao Tong University. The setup is schematically shown in Fig. 3. 


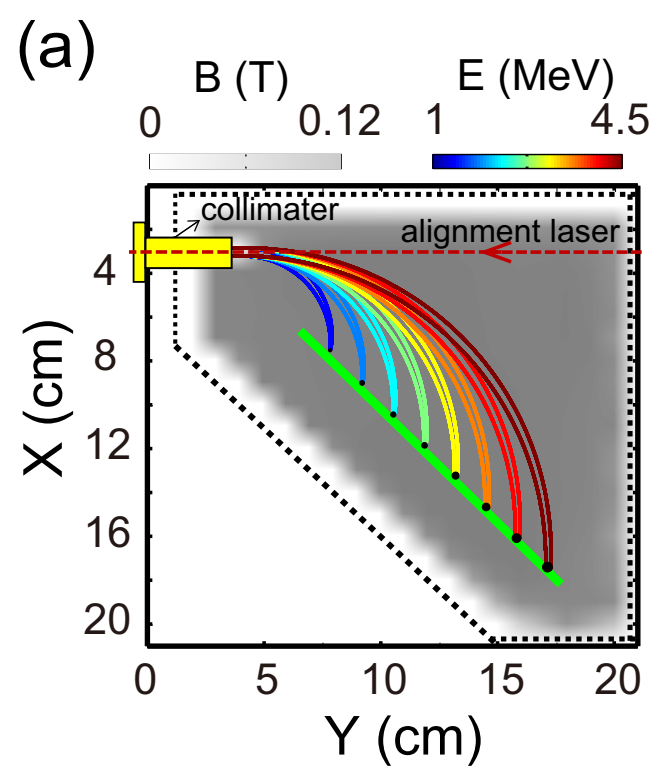

(b)

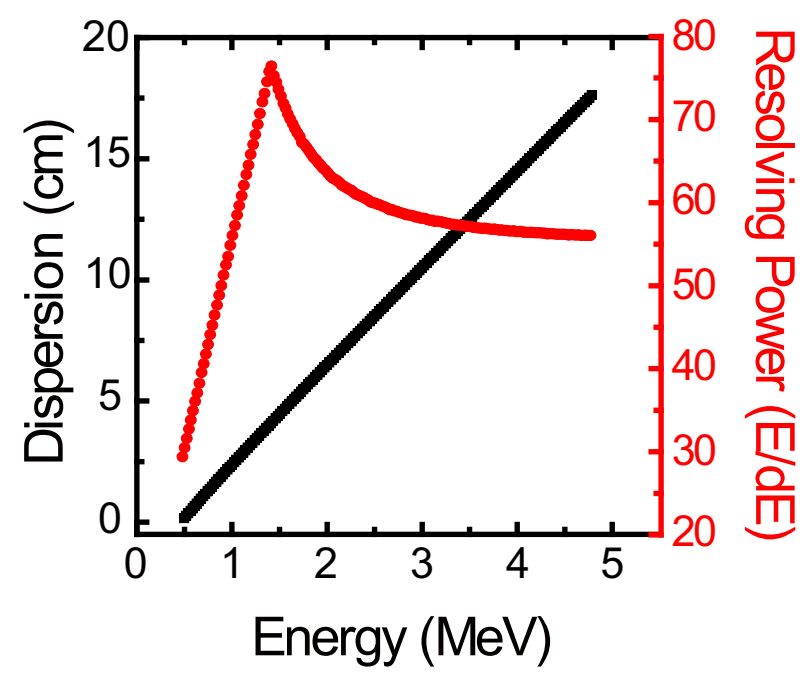

Figure 2: (color online) (a) Trajectories of electrons in magnetic field (gray area). Two colorbars depict the magnetic field distribution and the electron trajectories, respectively. Black circles show the electron beam sizes on the scintillator. (b) Dispersion and energy resolving power with respect to the electron energies. 


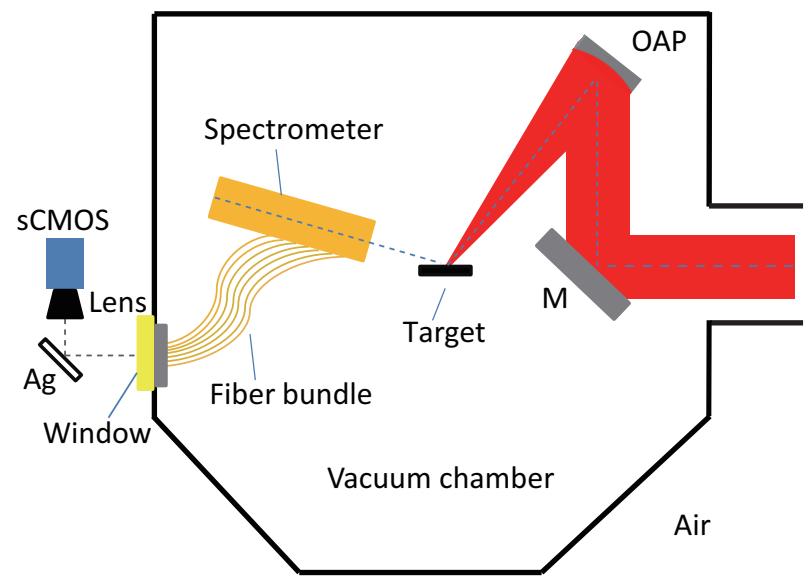

Figure 3: (color online) Schematic of the experimental setup. After reflected from the high reflective mirror $(\mathrm{M})$, the laser beam was focused by an off-axis parabola mirror (OAP) to the solid target. The electron spectrometer was placed in the target front side to measure the energy distribution of the fast electrons emitted close to the surface direction. The fluorescent light from the scintillator screen were guided by the fiber bundle and detected with a lenscoupled sCMOS imaging system. A silver mirror $(\mathrm{Ag})$ was used to avoid the $\gamma$-ray photons or high-energy particles directly bombarding the sCMOS sensor. 


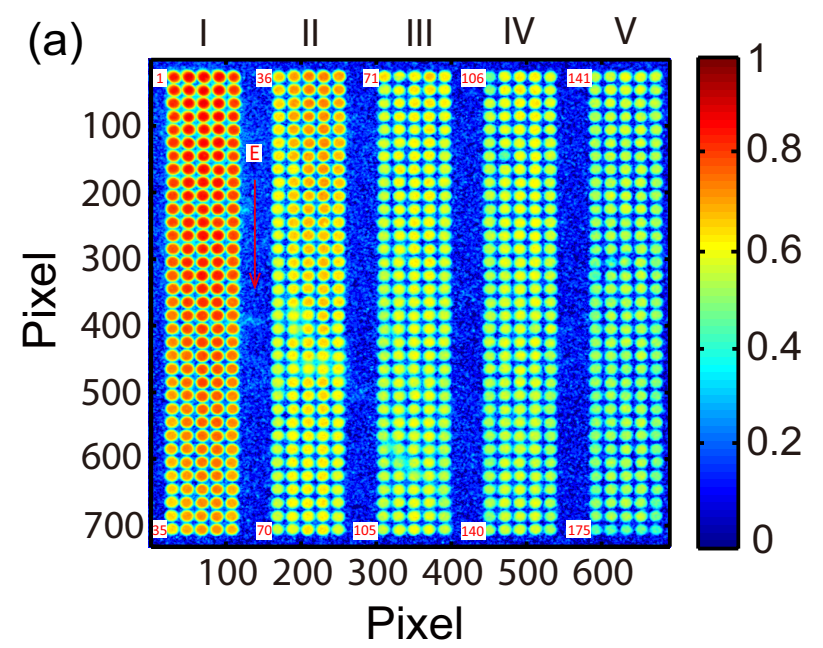

(b)

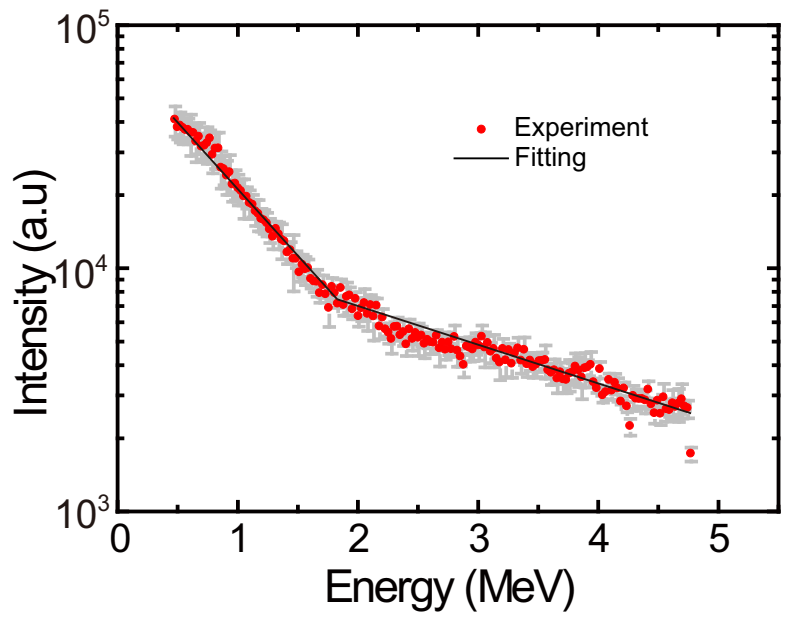

Figure 4: (color online) (a) Experimental raw data. Each colored circle spot corresponds to one fiber channel. The red numbers and arrow indicate the energy channel and energy increasing direction, respectively. (b) The measured light intensities (red) of the scintillatorbased detector and error bars (gray) for the fluctuations of different fibers in one energy channel. A fit of the experiment datas was also plotted by considering a two-temperature distribution of electrons. 
The arrangement of the fiber bundle can cause fluctuations of coupling efficiency between scintillator and fibers. To reduce the fluctuation in the data, the maximum and minimum values for each energy channel were removed. Taking

into account the energy resolutions, we calculated the electron spectra using the remaining three values of the fibers in each energy channel. The spectral distribution of the sampled fast electrons showing a two-temperature distribution is presented in Fig. 4(b) where the error bars are the data fluctuations from the remaining three values in the same energy channel.

The reliability and the on-line capability were also tested experimentally. 10 consecutive shots were taken in 3 minutes for the same laser and target parameters. The shot frequency was only determined by the time of replacing targets, which could be as short as a few 10's of seconds. Compared with the use of IPs, which need to vent the chamber to read the stored signals, the use of scintillator coupling with imaging system is much more attractive in high-repetition-rate experiments. One could obtain the data on electron energy spectrum shortly after each laser shot, which is the prerequisite to source optimization for applications. The shot-to-shot fluctuations of the spectra was measured to be less than 5\%, which is within the error bars shown in Fig. 4 .

\section{4. summary}

In summary, we have designed and tested a flexible, on-line electron spectrometer using a combination of plastic scintillator, plastic fiber array and sCMOS camera. This design would allow on-line measurement of fast electrons produced by high-repetition laser pulses. Better energy resolution can be obtained by using optical fibers with smaller diameters.

This spectrometer can also provide, though relatively modest, one-dimensional angular resolution from the five fiber channels perpendicular to the dispersion direction. The angular resolution could be improved by enlarging the entrance pinhole with a long slit and increasing the number of fiber channels in the plane perpendicular to the dispersion direction. With further revision, this novel 
design could be employed to measure the angular and energy distributions of low-divergence laser wakefield accelerated electron beams. Continuous improvement and development could facilitate many high-power laser-plasma research projects with a versatile diagnostic for fast electron generation.

215

\section{Acknowledgments}

We thank Professor Liming Chen for stimulating discussions and Professor Antonio Ting for improving the quality of manuscript. This work was supported by National Basic Research Program of China (Grant No. 2013CBA01502), National Natural Science Foundation of China (Grant Nos. 11421064, 11205100 (Grant No.2012YQ030142).

\section{References}

[1] M. Tabak, J. Hammer, M. E. Glinsky, W. L. Kruer, S. C. Wilks, J. Woodworth, E. M. Campbell, M. D. Perry, R. J. Mason, Ignition and high-gain with ultrapowerful lasers, Phys. Plasmas. 1 (1994) 1626-1634. doi:http://dx.doi.org/10.1063/1.870664.

[2] H. Daido, M. Nishiuchi, A. S. Pirozhkov, Review of laser-driven ion sources and their applications, Rep. Prog. Phys. 75 (5) (2012) 056401. doi:10.1088/0034-4885/75/5/056401.

[3] A. Macchi, M. Borghesi, M. Passoni, Ion acceleration by superintense laser-plasma interaction, Rev. Mod. Phys. 85 (2) (2013) 751-793. doi:10.1103/RevModPhys.85.751.

[4] L. M. Chen, M. Kando, M. H. Xu, Y. T. Li, J. Koga, M. Chen, H. Xu, X. H. Yuan, Q. L. Dong, Z. M. Sheng, S. V. Bulanov, Y. Kato, J. Zhang, T. Tajima, Study of x-ray emission enhancement via a high-contrast femtosecond laser interacting with a solid foil, Phys. Rev. Lett. 100 (2008) 045004. doi:https://doi.org/10.1103/PhysRevLett.100.045004. 
[5] G. Q. Liao, Y. T. Li, Y. H. Zhang, H. Liu, X. L. Ge, S. Yang, W. Q. Wei, X. H. Yuan, Y. Q. Deng, B. J. Zhu, Z. Zhang, W. M. Wang, Z. M. Sheng, L. M. Chen, X. Lu, J. L. Ma, X. Wang, J. Zhang, Demonstration of coherent terahertz transition radiation from relativistic laser-solid interactions, Phys. Rev. Lett. 116 (2016) 205003. doi:10.1103/PhysRevLett.116.205003.

[6] K. A. Tanaka, T. Yabuuchi, T. Sato, R. Kodama, Y. Kitagawa, T. Takahashi, T. Ikeda, Y. Honda, S. Okuda, Calibration of imaging plate for high energy electron spectrometer, Rev. Sci. Instrum. 76 (1) (2005) 013507. doi:10.1063/1.1824371.

[7] H. Chen, N. L. Back, T. Bartal, F. N. Beg, D. C. Eder, A. J. Link, A. G. MacPhee, Y. Ping, P. M. Song, A. Throop, L. Van Woerkom, Absolute calibration of image plates for electrons at energy between $100 \mathrm{kev}$ and 4 mev, Rev. Sci. Instrum. 79 (3) (2008) 033301. doi:10.1063/1.2885045.

[8] Z. Li, S. Nakamura, A. Fukumi, Y. Hayashi, S. Orimo, M. Nishiuchi, A. Sagisaka, M. Mori, T. Shirai, Y. Iwashita, A. Noda, H. Daido, Electron energy spectrometer for laser-driven energetic electron generation, Jpn. J. Appl. Phys. 44 (9A) (2005) 6796-6800. doi:10.1143/JJAP.44.6796.

[9] C. Gahn, G. D. Tsakiris, K. J. Witte, P. Thirolf, D. Habs, A novel 45-channel electron spectrometer for high intensity laserplasma interaction studies, Rev. Sci. Instrum. 71 (4) (2000) 1642. doi:http://dx.doi.org/10.1063/1.1150511.

[10] H. Chen, P. K. Patel, D. F. Price, B. K. Young, P. T. Springer, R. Berry, R. Booth, C. Bruns, D. Nelson, A compact electron spectrometer for hot electron measurement in pulsed laser solid interaction, Rev. Sci. Instrum. 74 (3) (2003) 1551. doi:http://dx.doi.org/10.1063/1.1526929.

[11] C. M. S. Sears, S. B. Cuevas, U. Schramm, K. Schmid, A. Buck, D. Habs, F. Krausz, L. Veisz, A high resolution, broad energy acceptance spectrom- 
eter for laser wakefield acceleration experiments, Rev. Sci. Instrum. 81 (7) (2010) 073304. doi:10.1063/1.3458013.

[12] H. J. Cha, I. W. Choi, H. T. Kim, I. J. Kim, K. H. Nam, T. M. Jeong, J. Lee, Absolute energy calibration for relativistic electron beams with pointing instability from a laser-plasma accelerator, Rev. Sci. Instrum. 83 (6) (2012) 063301. doi:10.1063/1.4725530.

[13] D. C. Eder, A. Throop, C. G. Brown, J. K. Jr., M. L. Stowell, D. A. White, P. Song, N. Back, A. MacPhee, H. Chen, W. DeHope, Y. Ping, B. Maddox, J. Lister, G. Pratt, T. Ma, Y. Tsui, M. Perkins, D. O'Brien, P. Patel, Mitigation of electromagnetic pulse (emp) effects from shortpulse lasers and fusion neutrons, LDRD Final Report. No. LLNL-TR411183.doi: $10.2172 / 950076$.

[14] H. Chen, S. C. Wilks, P. K. Patel, R. Shepherd, Short pulse laser produced energetic electron and positron measurements, Rev. Sci. Instrum. 77 (10) (2006) 10E703. doi:http://dx.doi.org/10.1063/1.2220141.

[15] T. Hosokai, K. Kinoshita, T. Ohkubo, A. Maekawa, M. Uesaka, A. Zhidkov, A. Yamazaki, H. Kotaki, M. Kando, K. Nakajima, S. V. Bulanov, P. Tomassini, A. Giulietti, D. Giulietti, Observation of strong correlation between quasimonoenergetic electron beam generation by laser wakefield and laser guiding inside a preplasma cavity, Phys. Rev. E. 73 (3) (2006) 036407. doi:https://doi.org/10.1103/PhysRevE.73.036407.

[16] Y. Glinec, J. Faure, A. Guemnie-Tafo, V. Malka, H. Monard, J. P. Larbre, V. D. Waele, J. L. Marignier, M. Mostafavi, Absolute calibration for a broad range single shot electron spectrometer, Rev. Sci. Instrum. 77 (10) (2006) 103301. doi:http://dx.doi.org/10.1063/1.2360988.

[17] K. Nakamura, B. Nagler, C. Tóth, C. G. R. Geddes, C. B. Schroeder, E. Esarey, W. P. Leemans, A. J. Gonsalves, S. M. 
Hooker, Gev electron beams from a centimeter-scale channel guided laser wakefield accelerator, Phys. Plasmas. 14 (5) (2007) 056708. doi:http://dx.doi.org/10.1063/1.2718524.

[18] K. Schmid, L. Veisz, F. Tavella, S. Benavides, R. Tautz, D. Herrmann, A. Buck, B. Hidding, A. Marcinkevicius, U. Schramm, M. Geissler, J. Meyer-ter Vehn, D. Habs, F. Krausz, Few-cycle laserdriven electron acceleration, Phys. Rev. Lett. 102 (12) (2009) 124801. doi:https://doi.org/10.1103/PhysRevLett.102.124801.

[19] J. S. Green, M. Borghesi, C. M. Brenner, D. C. Carroll, N. P. Dover, P. S. Foster, P. Gallegos, S. Green, D. Kirby, K. J. Kirkby, P. McKenna, M. J. Merchant, Z. Najmudin, C. A. J. Palmer, D. Parker, R. Prasad, K. E. Quinn, P. P. Rajeev, M. P. Read, L. Romagnani, J. Schreiber, M. J. V. Streeter, O. Tresca, C.-G. Wahlström, M. Zepf, D. Neely, Scintillator-based ion beam profiler for diagnosing laser-accelerated ion beams, Proc.SPIE 8079 (2011) 807919. doi:10.1117/12.888967.

[20] Y. C. Wu, B. Zhu, K. G. Dong, Y. H. Yan, Y. Q. Gu, Note: Absolute calibration of two drz phosphor screens using ultrashort electron bunch, Rev. Sci. Instrum. 83 (2) (2012) 026101. doi:http://dx.doi.org/10.1063/1.3681442.

[21] R. Kodama, K. Okada, Y. Kato, Development of a two-dimensional spaceresolved high speed sampling camera, Rev. Sci. Instrum. 70 (1) (1999) $625-628$. 